\title{
Faculty Development in Malta
}

Elizabeth Norris, Anne Marie Camilleri Podesta, Tanya Esposito, Simon Attard Montalto

Abstract

A faculty development program helps faculty members in their various roles as teachers, researchers and administrators and addresses their needs of professional development. Any hospital needs to consider how it will maintain continuity of the course itself and standards over time and maintain or increase its numbers of faculty. One should encourage ongoing support and enthusiasm within the organisation and plan for succession. This observational study illustrates how the education programme of the Malta Resuscitation Council (MRC) has achieved this goal to date and its plans for the future. We analyse the courses organised with reference to the success rate of candidates, the proportion of Instructor Potential (IP) candidates, their progression to full instructors and their contribution after that. The introduction of instructor days and the learning outcomes of the programme sequence is also illustrated.

Malta being a small country, served by one large hospital routinely invites guest overseas instructors. This helps to maintain standards, bring in new ideas, aid faculty development, ensure quality control of our instructors and prevent bias during assessments.

\section{Introduction of courses in Malta}

\section{MRC founded}

1999 PALS $\rightarrow 2005$ EPLS $\rightarrow 2015$ EPALS (European Paediatric Advanced Life Support)

2006 ETC (European Trauma Course)

2008 ALS (Advanced Life Support)

2009 GIC (Generic Instructor Course)

2010 ILS (Immediate Life Support)

2017 NLS (Neonatal Life Support)

2016 Annual Instructor Day
Educational support within courses - 'micro' level

National guidance on how the faculty should run each session was written for ALS and EPALS courses

A working faculty meal is held the evening before the course starts.

Online course report is written by the course director and relevant feedback acted on

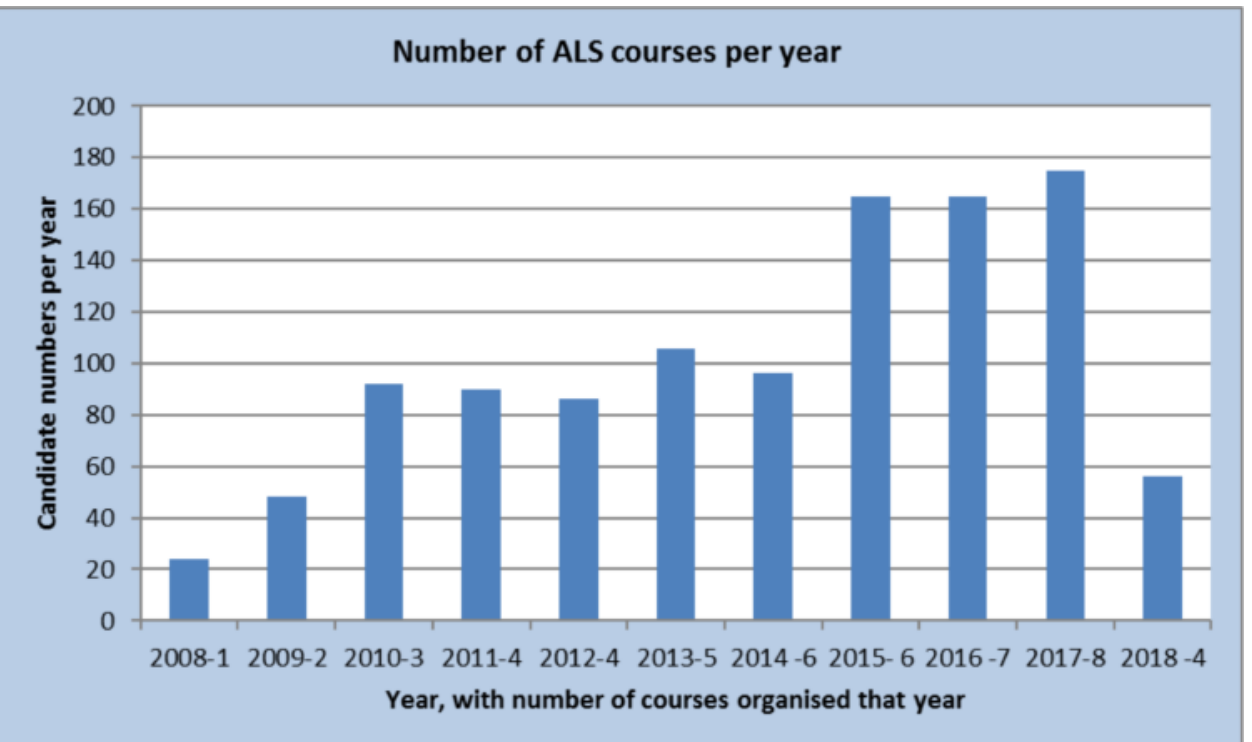

Table 1: This illustrates the numbers of courses per year and candidates taught

\section{EPALS}

Annual courses since 1999

19 courses organised to date

598 candidates

5 failures

1 to 2 IPs per course

20 active EPALS instructors and 6 ICs

\section{NLS}

Courses since 2017

2 NLS courses to date

48 candidates

4 IPs

3 Maltese NLS instructors of which 2 have already co-directed

\section{Instructor Days}

To date MRC has offered annual instructor days since 2016 at no charge.
The MRC introduced annual instructor days:

$\rightarrow$ to refresh teaching theory

raise awareness of novel thinking in medical education

encourage and motivate young instructors to help support future faculty development $>$ encourage involvement with the MRC.

\section{Instructor Day}

$>$ refresh and update knowledge of 2015 international resuscitation guidelines

introduction of ALSi simulators to improve our simulation platform

Dr Carsten Lott (ICC for ALS) and Dr Joachim Schlieber (ICC for ILS) were involved in the first instructor day.

The second instructor day was planned by ERC educators Elizabeth Norris and Anne Marie Camilleri Podesta:

\section{Instructor Day}

Clinical teaching

Update on Acute Coronary Syndromes

Small group teaching

Giving effective feedback

\section{Instructor Day}

Review of first NLS course

Simulation teaching - helping candidates

immerse in simulation and embed Non Technical skills

Feedback - Using the Learning Conversation

Coaching - Coping with difficult feedback

Getting assessment right

In 2018 the MRC organised the first Director's day and all active Directors attended.

\section{Discussion}

There is considerable investment in developing instructors, both financially and in time. A longer term mentorship programme could encourage these individuals to continue with training and ensure there are opportunities to teach as an IC early before enthusiasm is lost.

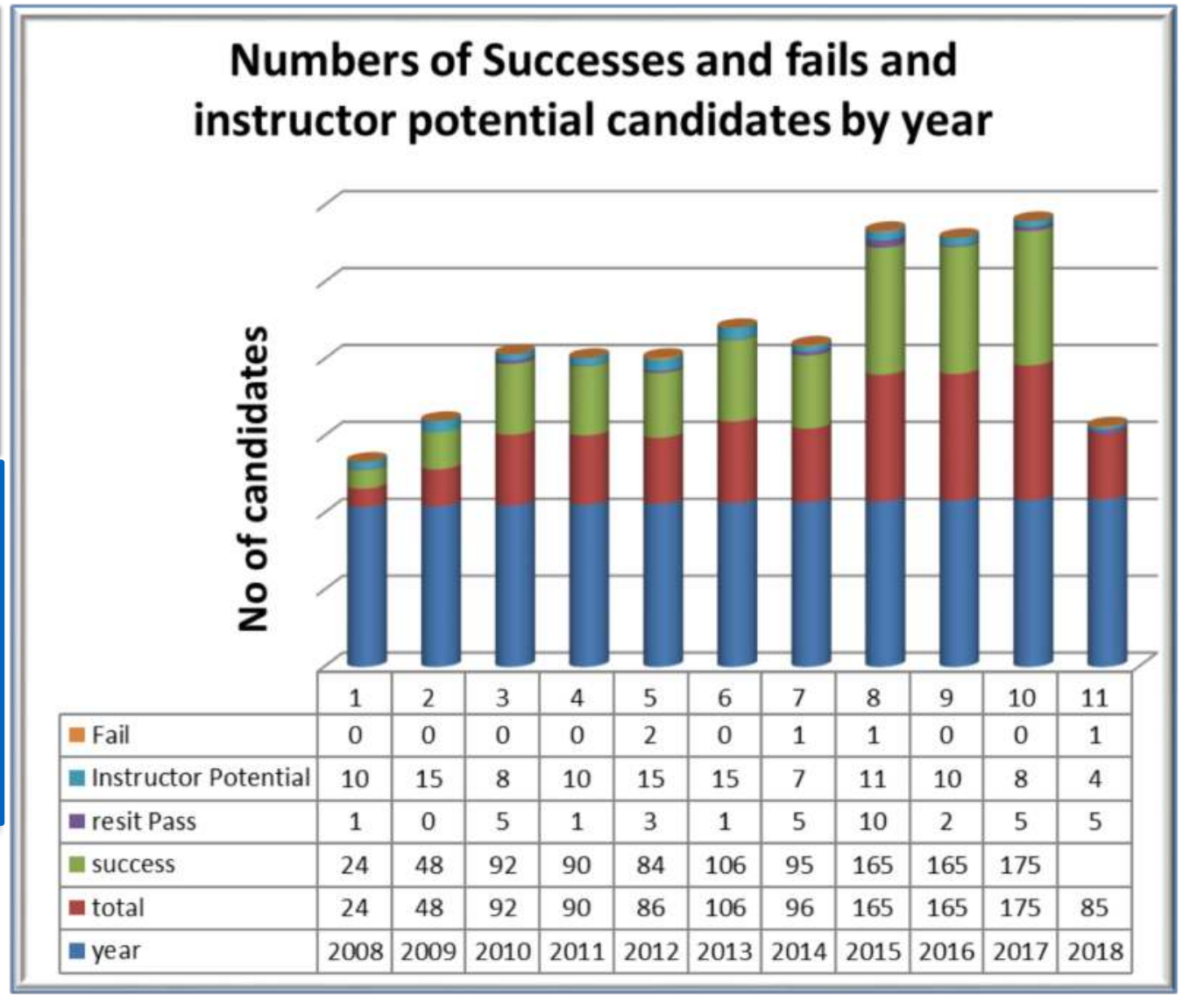

Table 2 (above)

No. of success / failures \& no. of IPs per year:

Since 2016, the percentage of foundation yea doctors attending ALS courses has increased. This has led to lower IP rates as usually it is doctors with Core Medical training or Speciality training who are given IP status.

\section{Conclusion}

The MRC has taken an exemplar approach to the development and continuity of resuscitation teaching, development and support of faculty which may form a useful model for others to follow. It has considered problems at both the micro level of running courses with adequate equipment, preparation and support of junior faculty and at national level, namely a high standard of readily available courses, adequate faculty which is prevented from becoming insular by the regular invitation of outside faculty, regular instructor free training days and a very active resuscitation council 